\title{
Gene Knock Up via 3'UTR editing to study gene function in
}

\section{vivo}

\section{Authors and affiliations}

Kärt Mätlik ${ }^{1,8 *}$, Soophie Olfat ${ }^{1,2}$, Daniel R. Garton ${ }^{1}$, Ana Montaño-Rodriguez ${ }^{1}$, Giorgio Turconi ${ }^{1}$, L. Lauriina Porokuokka $^{1}$, Anne Panhelainen ${ }^{3}$, Nadine Schweizer ${ }^{2}$, Jaakko Kopra ${ }^{4}$, Mark C. Cowlishaw ${ }^{1}$, T. Petteri Piepponen ${ }^{4}$, Fu-Ping Zhang ${ }^{5,6}$, Petra Sipilä5 ${ }^{5}$ Johan Jakobsson $^{7}$, Jaan-Olle Andressoo ${ }^{1,2}$ *

${ }^{1}$ Department of Pharmacology, Faculty of Medicine \& Helsinki Institute of Life Science, University of Helsinki, 00290 Helsinki, Finland

${ }^{2}$ Division of Neurogeriatrics, Department of Neurobiology, Care Sciences and Society (NVS), Karolinska Institutet, 17177 Stockholm, Sweden

${ }^{3}$ Institute of Biotechnology, University of Helsinki, 00014 Helsinki, Finland

${ }^{4}$ Division of Pharmacology and Pharmacotherapy, Faculty of Pharmacy, University of Helsinki, 00014 Helsinki, Finland

${ }^{5}$ Research Centre for Integrative Physiology and Pharmacology, Institute of Biomedicine and Turku Center for Disease Modeling, University of Turku, 20520 Turku, Finland

${ }^{6}$ Biomedicum Stem Cell Center, University of Helsinki, 00290 Helsinki, Finland

${ }^{7}$ Laboratory of Molecular Neurogenetics, Department of Experimental Medical Science, Wallenberg Neuroscience Center and Lund Stem Cell Center, BMC A11, Lund University, 22184 Lund, Sweden

${ }^{8}$ Current address: Laboratory of Developmental Neurobiology, The Rockefeller University, 10065 New York, NY, USA

* Corresponding authors 


\section{Abstract}

Currently available genetic tools do not allow researchers to upregulate ('Knock Up') the levels of a given protein while retaining its cell-type-specific regulation. As a result, we have limited ability to develop overexpression-related disease models, to study the contribution of single genes in diseases caused by copy number variations and to identify disease pathways for drug targets. Here we develop two approaches for endogenous gene upregulation: conditional Knock $\underline{\mathrm{Up}}$ (cKU) utilizing the Cre/lox system, and CRISPR-Cas9 mediated gene Knock $\underline{\text { Up }}(\mathrm{KU})$ in wild-type mouse embryos and human cells. Using glial cell line derived neurotrophic factor (GDNF) as a proof of concept, we show that both approaches resulted in upregulation of endogenous GDNF levels without disturbing $G d n f$ 's expression pattern. Furthermore, CNS-specific GDNF cKU resulted in dopaminergic abnormalities and schizophrenia-like phenotypes. Our results suggest that gene Knock Up can reveal unknown gene functions and provide novel entry points for studying neurological disease. 


\section{Background}

Understanding how aberrations in gene functioning lead to diseases and how the activity of endogenous signalling pathways can be harnessed to lead to different phenotypic outcomes and therapies is one of the major challenges for biomedical research and drug industry. One of the obstacles along this path is our limited ability to upregulate specific genes in endogenous signalling pathways—either by genetic tools or pharmacological compounds- to study gene function and to identify the genes or pathways that are beneficial if upregulated in a disease. Knocking out a gene or reducing the activity of the encoded protein is relatively achievable with various genetic tools. However, increasing the expression of specific genes while maintaining their natural expression pattern is much more of a challenge. Although transgenic animals and viral overexpression methods are widely used for this purpose, they usually result in gene expression levels that far exceed that of endogenous genes, and importantly, transgene expression patterns do not usually follow the expression of native genes ${ }^{1}$. Yet, human studies have shown that even as little as a 50-100\% increase in endogenous gene expression levels or activity is sufficient to cause neurological disease $\mathrm{e}^{2-5}$. Importantly, many of the genes implicated in these diseases encode regulatory proteins with restricted spatiotemporal expression patterns, further limiting the use of transgenic overexpression tools. Therefore, a tool that would allow researchers to conditionally increase gene expression levels within a physiological range, while retaining the correct expression pattern and transcriptional regulation, would be useful in many fields of basic and biomedical research, as well as for drug development.

With this in mind, we reasoned that in order to maintain correct gene expression patterns, modifications should be targeted towards regions that regulate expression post-transcriptionally, i.e., at the level of mRNA or protein. We focused on the 3' untranslated region (3'UTR), because this region is well known for its role in regulating mRNA stability ${ }^{6}$. We hypothesised that by conditionally replacing negative 
regulation elements residing in the 3'UTR we could 'Knock Up' endogenous gene expression levels without altering the gene's spatiotemporal expression pattern. For a proof of concept, we generated a conditional Knock $\underline{\mathrm{U}} \mathrm{p}(\mathrm{cKU})$ model for the neurotrophic factor GDNF (glial cell line-derived neurotrophic factor), whose levels we have previously shown to be negatively regulated through its $3^{1}$ UTR in vitro and in vivo ${ }^{7}$ GDNF is critically important for the development of peripheral tissues, including the kidney ${ }^{8-11}$. In addition, GDNF is a potent neurotrophic factor for midbrain dopamine neurons in culture ${ }^{12}$ and promotes dopamine synthesis and dopaminergic neuron fibre outgrowth if applied ectopically into the brain ${ }^{13}$. Increased dopamine signalling is associated with different neuropsychiatric diseases, including schizophrenia, where increased levels of presynaptic dopamine in the striatum are commonly observed ${ }^{14-16}$. Therefore, we were particularly interested in the outcome of brain-specific elevation of endogenous GDNF levels on brain dopamine system function, and the potential relevance of altered dopamine signalling in the context of schizophrenia.

To be able to specifically increase GDNF expression in the brain, we utilized the FLEx cassette ${ }^{17}$ to conditionally replace the Gdnf 3'UTR with a 3'UTR and polyadenylation signal derived from the bovine growth hormone (bGHpA), using Cre-mediated recombination. We found increased GDNF expression in GDNF cKU mice, while Gdnf expression sites appeared unchanged. Mice with endogenous GDNF upregulation in the central nervous system (CNS) exhibited increased presynaptic dopamine signalling in the striatum, and reduced dopamine levels and altered gene expression in prefrontal cortex, resembling findings from schizophrenic patients and animal models ${ }^{14,15,18,19}$. Interestingly, we found that reduction in prefrontal cortex dopamine levels can also be triggered by striatal upregulation of endogenous GDNF in adult mice using striatal AAV-Cre delivery. Finally, we asked if endogenous gene expression levels can be elevated in wild-type cells by 3'UTR editing. We found that excising inhibitory regions from the Gdnf 3'UTR using the CRISPR-Cas9 system increased 
endogenous GDNF expression levels in wild-type mouse embryos and in human cells. Taken together, our results suggest that gene Knock Up via 3'UTR editing is applicable to human cells and wild-type mice and can reveal novel information about gene function.

\section{Results}

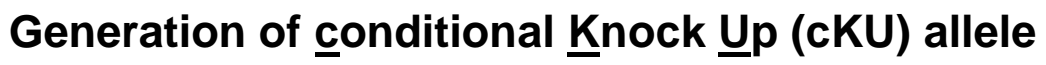

Gene overexpression levels and pattern in transgenic animals are mainly dependent upon transgene copy number, specificity of used promoter, integration site, and epigenetic changes across generations. As a result, the extent of overexpression and expression pattern of transgenes usually differ significantly from those of endogenous gene products ${ }^{1}$. Therefore, it can be difficult to draw conclusions about the functions of proteins, whose levels and expression pattern are tightly controlled. We hypothesised that by conditionally preventing post-transcriptional negative regulation of mRNA levels by 3'UTR replacement, endogenous mRNA and protein levels could be increased without changing the gene's spatiotemporal expression pattern (Fig. 1a). We further hypothesised that by utilizing the Cre/lox system, an increase in endogenous gene levels could also be restricted to specific tissues or cell types. Notably, by only affecting post-transcriptional regulation, we expected that gene expression would not be induced in cells that do not normally express the gene (Fig. 1a). 
a

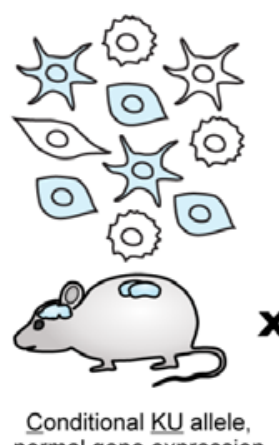
normal gene expression

b

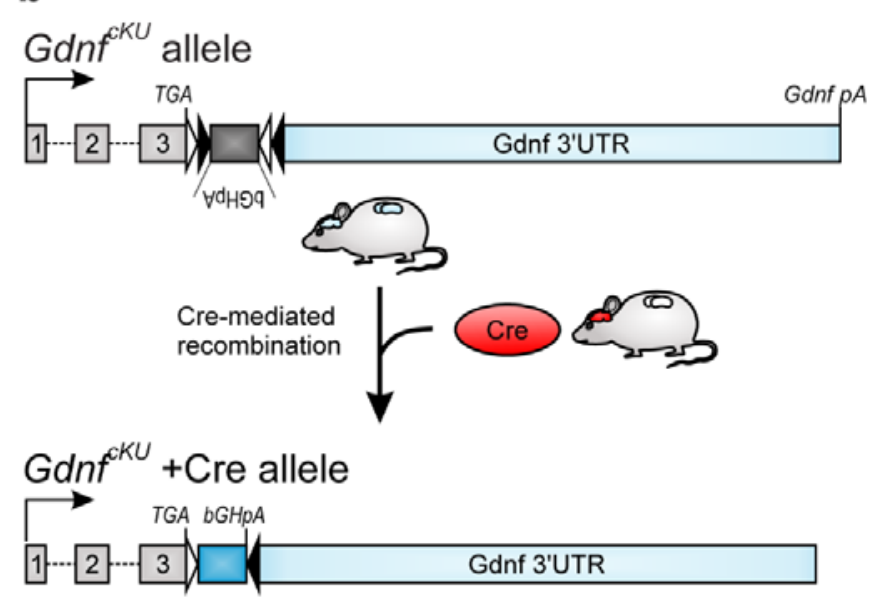

Transcription 」

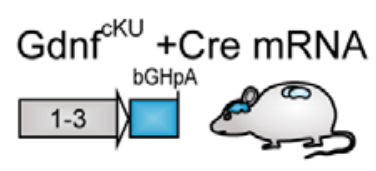

Figure 1 Schematic of conditional Knock $\underline{\mathrm{U}} \mathrm{p}(\mathrm{cKU})$ approach. a Principle of conditional Knock Up of endogenous gene expression in natively expressing cells using the $\mathrm{cKU}$ allele combined with the Cre/lox system. b Schematic of $G d n f^{C U}$ allele (top panel) and the resulting mRNA carrying a short 3'UTR (bottom panel) devoid of miR binding sites.
To provide a proof of concept for the

approach, we generated a conditional Knock

$\underline{\mathrm{U} p}(\mathrm{cKU})$ allele for glial cell line-derived

neurotrophic factor (GDNF) by inserting a

FLEx cassette $^{17}$ containing bGHpA—a 3'UTR

sequence previously shown to lead to high

expression from the cognate $\mathrm{mRNA}^{20}$ — in an

inverted orientation immediately downstream

of the stop codon of the Gdnf gene (Fig. 1b,

Extended Data Fig. 1a). To evaluate the extent and expression pattern of conditional GDNF

overexpression in $G d n f^{c K U}$ mice, we first

analysed the kidneys, where $G d n f$ 's expression

pattern and the outcomes of Gdnf gene

deletion and increased levels of endogenous

GDNF have been well characterized. Mice

lacking GDNF have no kidneys ${ }^{8-11}$, whereas

endogenous GDNF overexpression in GDNF

hypermorphic mice causes a severe reduction

in kidney size due to reduced migration of progenitors from the ureteric bud tip to the ureteric bud $\operatorname{trunk}^{21}$, and other histological alterations ${ }^{7}$. We first crossed $G d n f^{\epsilon K U}$ mice to a Pgk1-Cre mouse line, where Cre expression results in an early and uniform recombination ${ }^{22}$. We found that Gdnf mRNA and GDNF protein levels were significantly increased in the kidneys of $G d n f^{W T / c K U}$;Pgk1-Cre mice compared to wild-type mice at postnatal day (P) 3 (Fig. 2a-b). Similar to what we previously observed 
in GDNF hypermorphs ${ }^{7}$, kidney size was severely reduced in mice expressing increased levels of GDNF (Fig. 2c).
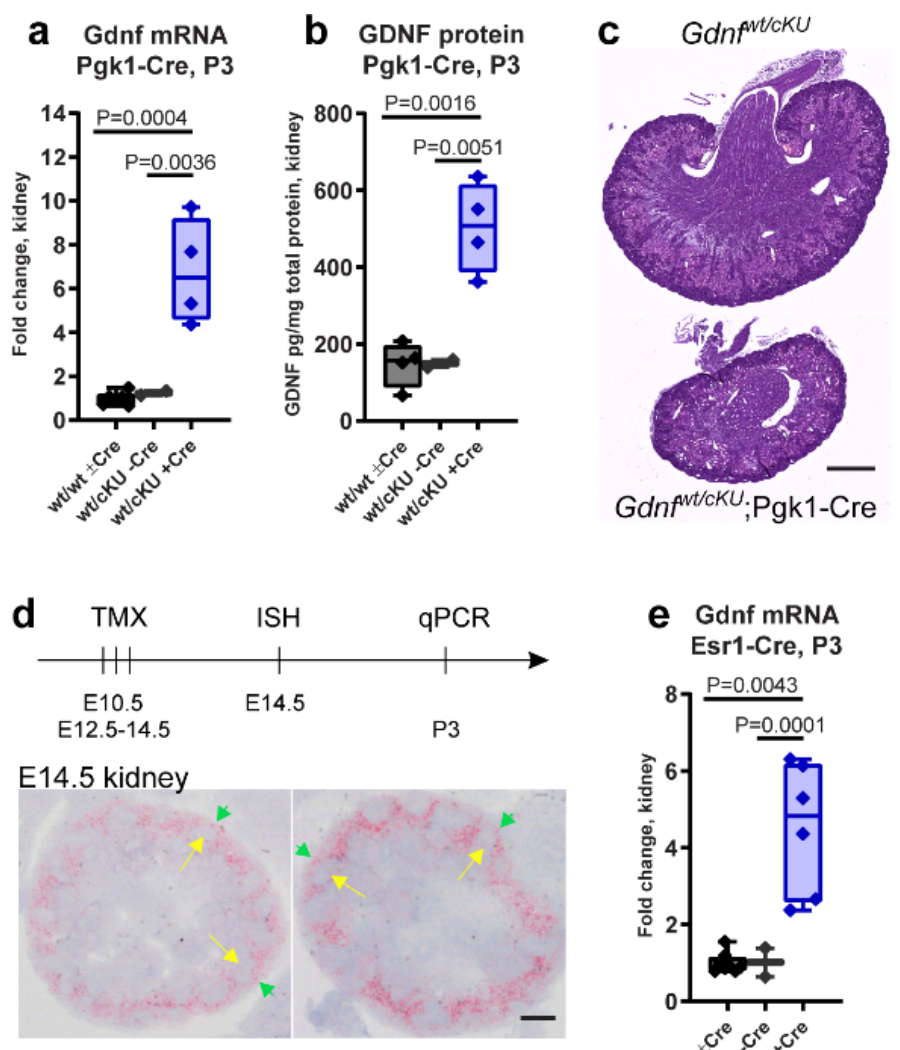

Next, we crossed $G d n f^{\epsilon U}$ mice to a tamoxifen-inducible Esr1-Cre line ${ }^{23}$ and administered tamoxifen to pregnant females at E10.5. At E14.5, in situ hybridization using RNAscope probes against Gdnf revealed a stronger signal in $G d n f^{w t / c K U}$;Esr1e Gdnf mRNA

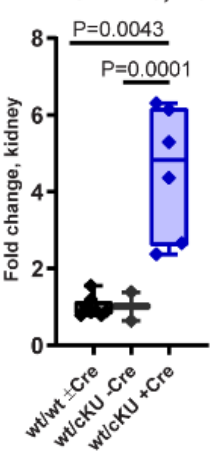

Cre mice compared to wild-type littermates, whereas Gdnf's expression pattern in these mice was normal (Fig. 2d). To measure Gdnf mRNA levels, tamoxifen was then injected on three consecutive days at E12.5-E14.5, and mice were dissected at P3. We found that

Figure 2 Endogenous GDNF in the kidney. a-b Gdnf mRNA (a) and protein (b) levels in P3 kidneys. c Kidney hematoxylineosin staining at P3. d Timeline of tamoxifen injection (top panel) and representative in situ hybridization image (bottom panel) from E14.5 kidney. Green arrows indicate GDNFexpressing metanephric mesenchyme and yellow arrows indicate ureteric buds. e Gdnf mRNA expression in P3 kidneys after tamoxifen injection. Median, upper and lower quartiles, and maximum and minimum values are shown. Scale bar 500 $\mu \mathrm{m}$ (c) or $100 \mu \mathrm{m}$ (d). See Methods for details.

Gdnf expression in the kidneys of $G d n f^{W T / c K U} ;$ Esr1-Cre mice was increased by more than 4-fold compared to wild-type and $G d n f^{W T / c K U}$ controls (Fig. 2e). Therefore, we conclude that the $G d n f^{c K U}$ allele allows us to increase endogenous $G d n f$ expression at different stages of development, and, in line with our hypothesis, does not change normal $G d n f$ expression pattern. 


\section{$G d n f^{c K U}$ mice have schizophrenia-like features}

We next crossed the $G d n f^{c K U}$ mice to a Nestin-Cre-expressing mouse line ${ }^{24}$ to restrict the increase in endogenous GDNF levels to cells expressing GDNF in the CNS from E11.5 onwards. In the adult nigrostriatal system, GDNF is mostly produced in a sparse population of striatal interneurons ${ }^{25}$ that

a Gdnf mRNA

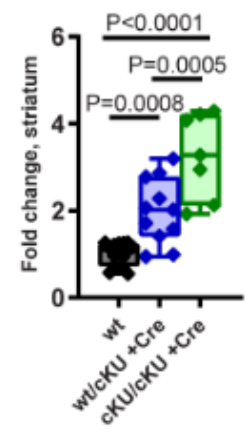

b Gdnfow' ; Nestin-Cre

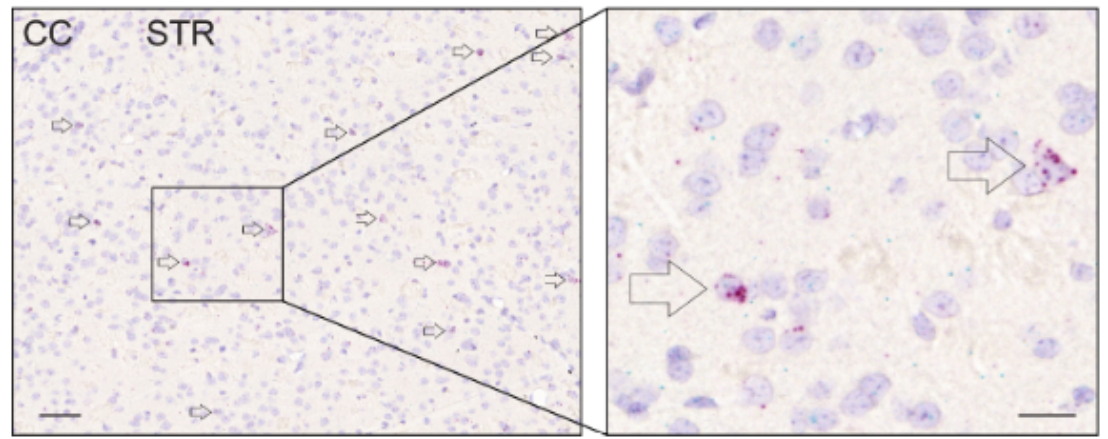

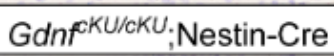

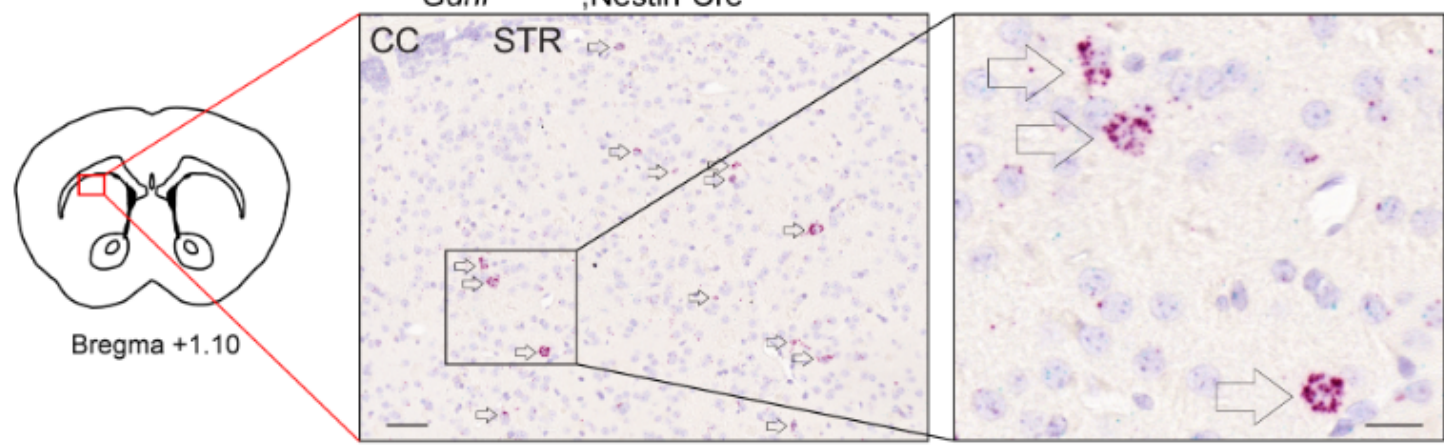

C Dopamine striatum

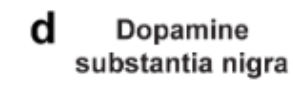

e

DA release, STR

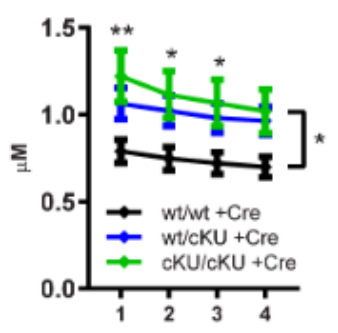

f

DA clearance
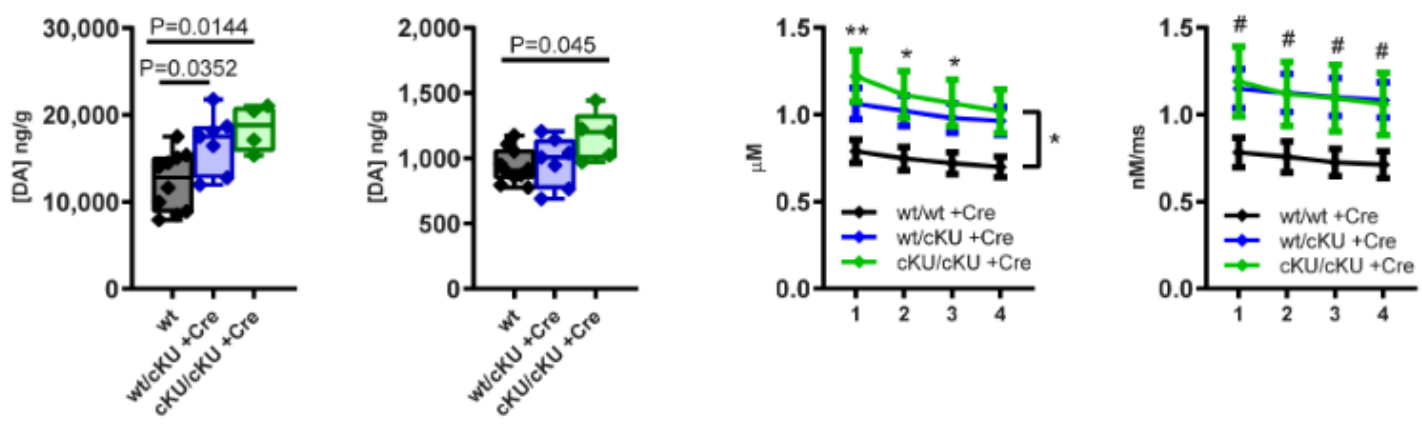

Figure 3 Endogenous GDNF in the brain of adult $G d n f^{c K}$; Nestin-Cre mice. a-b Gdnf mRNA levels (a) and expression pattern revealed by RNAscope probes (b) in the striatum. c-d Total tissue dopamine levels in the striatum (c) and SN (d). e-f Stimulated dopamine release (e) and re-uptake (f) in striatal slices measured with CV. a, c-d Median, upper and lower quartiles, and maximum and minimum values are shown. e-f Error bars denote mean \pm SE. Scale bar $50 \mu \mathrm{m}(b$, left panel) or $10 \mu \mathrm{m}$ (b, right panel). See Methods for details. 
regulates striatal output ${ }^{26}$. We analysed $G d n f$ expression in the striatum of $G d n f^{c K U}$;Nestin-Cre mice and found that both Gdnf mRNA and GDNF protein levels were increased in $G d n f^{c K U}$ mice (Fig. 3a and Extended Data Fig. 2a), whereas Gdnf's expression pattern appeared normal (Fig. 3b). In line with previous findings ${ }^{7}, G d n f^{K U}$; Nestin-Cre mice exhibited increased levels of total tissue dopamine in the striatum and substantia nigra (SN) (Fig. 3c-d) and increased evoked dopamine release and uptake in the striatum (Fig. 3e-f).

Increased striatal dopamine signalling, particularly in the dorsal striatum, is a hallmark of schizophrenia $^{14-16}$. Furthermore, studies on schizophrenic patients and a mouse model overexpressing dopamine receptor D2 (Drd2) have suggested that increased dopamine signalling in the striatum results in reduced dopamine signalling in the prefrontal cortex $(\mathrm{PFC})^{18,19}$. We therefore asked if increased dopamine signalling in the striatum of $G d n f^{c K U}$; Nestin-Cre mice alters dopamine levels in the prefrontal cortex. Indeed, we found that dopamine levels were markedly reduced in the PFC of $G d n f^{w t / c K U}$;NestinCre and $G d n f^{c K U / c K U}$; Nestin-Cre mice at two months of age (Fig. 4a), indicating that an increase in presynaptic dopamine in the nigrostriatal system also leads to aberrant dopamine signalling in the PFC in our mouse model.

Studies on patients with schizophrenia have identified several changes in gene expression in the PFC. In particular, reduced $\operatorname{Drd} 2$ expression in the PFC is associated with schizophrenia ${ }^{27-30}$. We found that Drd2 mRNA levels were significantly reduced in the PFC of $G d n f^{w t / c K U}$;Nestin-Cre (heterozygous) mice and $G d n f^{c K U / c K U}$;Nestin-Cre (homozygous) mice (Fig. 4b). To obtain a more in-depth understanding of gene expression changes in the PFC, we performed RNA sequencing (Fig. 4c). We identified 311 differentially expressed (DE) genes in $G d n f^{w t / c K U}$;Nestin-Cre mice and 214 genes in $G d n f^{c K U / c K U}$;Nestin-Cre mice (Extended Data Fig. 2b-d). Functional annotation highlighted genes belonging to Gene Ontology (GO) categories related to dopamine signalling and myelination as most 


\section{a}

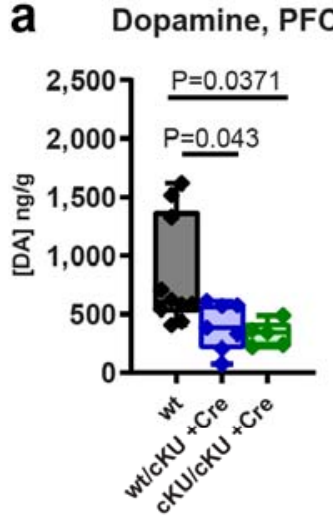

b Drd2 mRNA, PFC

\section{e}
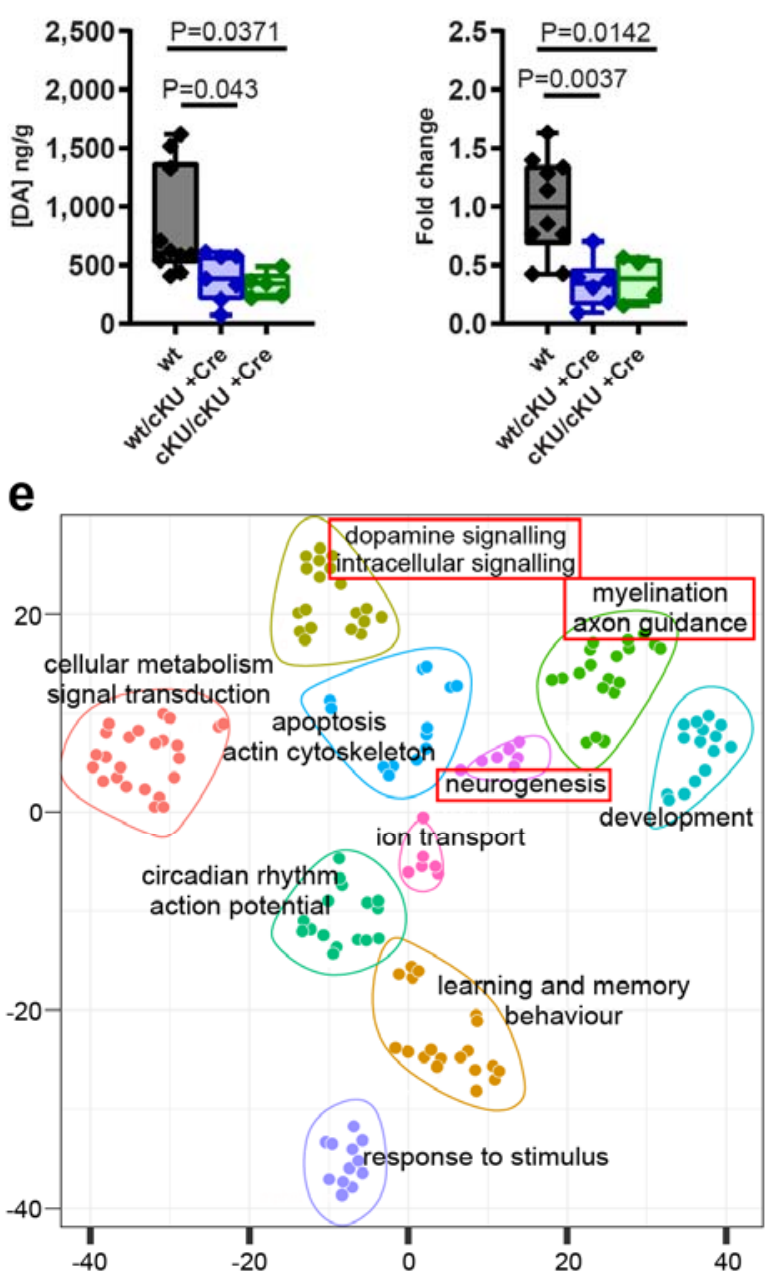

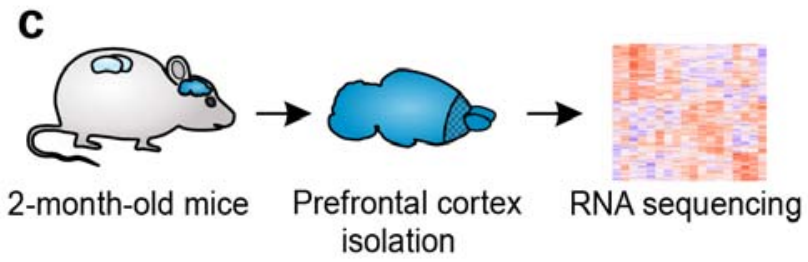

d

\begin{tabular}{|llr|}
\hline GO ID & Term & p-value \\
\hline GO:0007212 & dopamine receptor signaling pathway & $<0.0001$ \\
\hline GO:0001963 & synaptic transmission, dopaminergic & $<0.0001$ \\
\hline GO:0001975 response to amphetamine & $<0.0001$ \\
\hline GO:0022010 & central nervous system myelination & 0,00011 \\
\hline GO:0042552 & myelination & 0,00013 \\
\hline
\end{tabular}

f DE genes

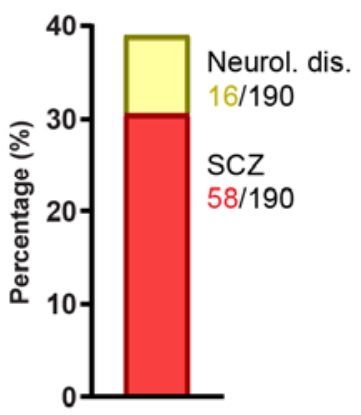

9 Pre-pulse inhibition

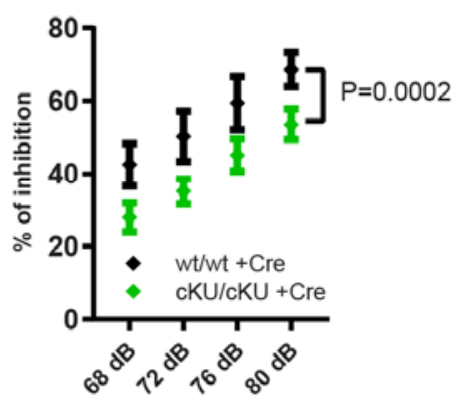

Figure 4 Schizophrenia-related phenotypes in $G d n f^{K U}$;Nestin-Cre mice. a Dopamine levels in the PFC. b Drd2 mRNA levels. c Schematic of RNA sequencing experiment. d Enriched GO categories in $G d n f^{f K U / c K U}$;Nestin-Cre (homozygous) mice. e Scatter plot of GO enrichment analysis in $G d n f^{K U / c K U}$;Nestin-Cre mice. Gene ontology categories most associated with schizophrenia are highlighted with red boxes. $\mathbf{f}$ Proportion of DE genes associated with schizophrenia or other neurodevelopmental disorders. g Pre-pulse inhibition test. GO, Gene Ontology. a-b Median, upper and lower quartiles, and maximum and minimum values are shown. $\mathbf{g}$ Error bars denote mean \pm SE. See Methods for details.

enriched among DE genes (Fig. 4d, Extended Data Fig. 2e). Further analysis on the top 150 enriched

GO categories revealed 10 distinct clusters of DE genes, mostly related to nervous system development and function (Fig. 4e). Moreover, we found that many of the DE genes have previously been associated with schizophrenia in either genome-wide association studies or based on gene expression changes in schizophrenic patients (Fig. 4f and Extended Data Table 1), suggesting that the dysregulated pathways in $G d n f^{w / t c K U}$;Nestin-Cre and $G d n f^{C K U / c K U}$;Nestin-Cre mice share similarities with schizophrenia. 
To investigate the behavioural outcome of aberrant dopamine signalling and gene expression in the PFC of $G d n f^{c K U / c K U}$; Nestin-Cre mice, we used the pre-pulse inhibition (PPI) test, which reflects the ability to successfully integrate and inhibit sensory information. Patients with schizophrenia and animal models with schizophrenia-like features have diminished responses to $\mathrm{PPI}^{18,19,31-33}$, which is believed to reflect impaired striatal and cortical dopamine transmission ${ }^{32-34}$. We found that the extent of PPI was significantly reduced in $G d n f^{c^{K U / c K U}}$;Nestin-Cre mice (Fig. 4g), suggesting that altered dopamine signalling is paralleled by a deficit in sensorimotor gating. Therefore, we conclude that a tissue-specific increase in endogenous GDNF expression in the CNS at midgestation results in a phenotypic resemblance to schizophrenic patients, suggested by increased presynaptic dopamine signalling in the striatum, reduced dopamine levels in the PFC, reduced expression of several genes and pathways identified in post-mortem samples from the PFC of schizophrenic patients, and a deficit in PPI, a known endophenotype of schizophrenia (Table 1).

\section{Adult-onset GDNF cKU affects PFC DA system}

Given that schizophrenia symptoms usually start in adolescence or adulthood, we were interested in whether the schizophrenia-like features found in $G d n f^{w t / c K U}$;Nestin-Cre and $G d n f^{c K U / c K U}$;Nestin-Cre mice could also be induced upon an adult-onset increase of endogenous GDNF expression. To that end, we injected AAV-Cre bilaterally into the striata of adult $G d n f^{v t / c K U}$ and $G d n f^{c K U / c K U}$ mice (Fig. 5a). We found that 2 months after AAV-Cre injection Gdnf expression was increased by more than 2-fold (Fig. 5b), resulting in an increase in dopamine release in response to a burst stimulus (Extended Data Fig. 3a). At the same time, total levels of striatal dopamine were not significantly increased, and evoked dopamine release and uptake were not affected (Extended Data Fig. 3b-d). However, dopamine levels in the PFC were significantly reduced by $\sim 50 \%$ (Fig. 5c), suggesting that changes in PFC dopamine 
signalling can be triggered already by 2 -fold increase in endogenous GDNF levels in the adult striatum and by relatively minor changes in striatal dopamine function. We also administered the PPI test but found no indication of deficits in sensorimotor gating at the tested time point (Fig. 5d).

\begin{tabular}{|c|c|c|c|}
\hline Phenotype & $\begin{array}{l}G d n f^{c K U / c K U} \\
\text { Nestin-Cre }\end{array}$ & $\begin{array}{l}\text { Gdnf } \\
\text { AAVU/cKU } \\
\text { Ane }\end{array}$ & $\begin{array}{l}\text { Findings in } \\
\text { humans }\end{array}$ \\
\hline Increased striatal DA levels & + & $+/-$ & +15 \\
\hline Reduced prefrontal cortex DA levels & + & + & +27 \\
\hline $\begin{array}{l}\text { Reduced PFC dopamine D2 receptor } \\
\text { levels }\end{array}$ & + & n.a & $+{ }^{27-30}$ \\
\hline Deficit in pre-pulse inhibition & + & - & $+35,36$ \\
\hline
\end{tabular}

Table 1 Phenotypes of $G d n f^{K U / c K U}$;Nestin-Cre mice that resemble findings from patients with schizophrenia.

Taken together, we propose that $G d n f^{\kappa U}$ mice may present a useful tool for studying the pathologies and treatments arising from increased presynaptic striatal dopamine function, as well as for finding therapeutic strategies that specifically target this deficit in schizophrenia. The ability to upregulate endogenous gene expression in developing, adolescent and adult animals may also allow researchers to investigate the importance of timing the onset of altered protein levels, which could have relevance for certain nervous system diseases ${ }^{37,38}$.
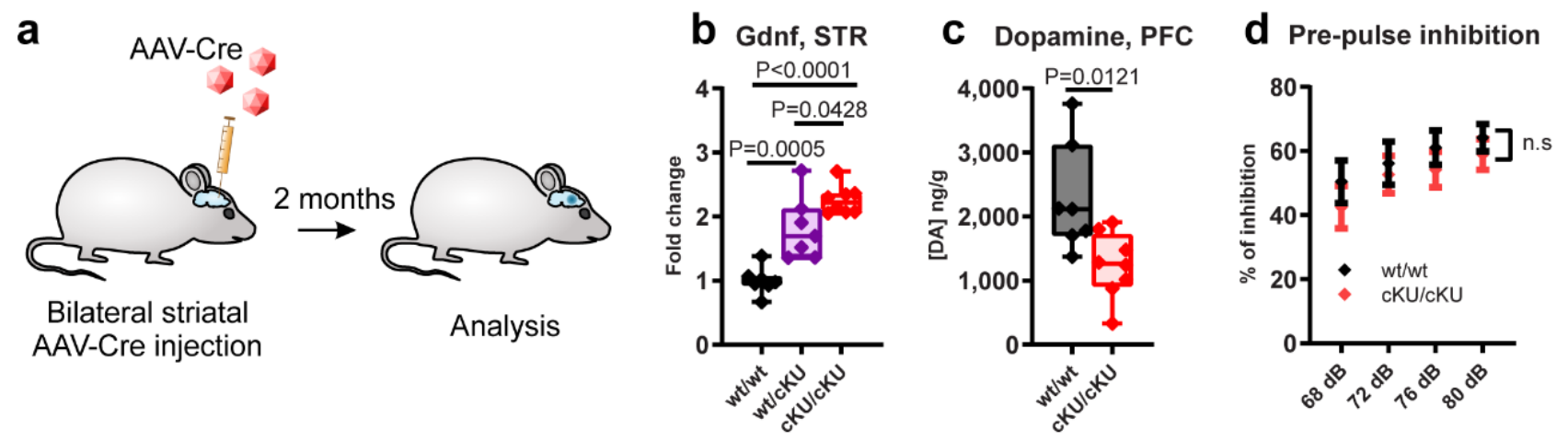

Figure 5 Dopamine system in $G d n f^{K U}$ mice after bilateral striatal AAV-Cre injection. a Experiment schematic. b Striatal Gdnf levels 2 months after AAV-Cre. c Total levels of dopamine in the PFC. d Pre-pulse inhibition test. b-c Median, upper and lower quartiles, and maximum and minimum values are shown. $\mathbf{d}$ Error bars denote mean \pm SE See Methods for details. 


\section{3'UTR editing with CRISPR-Cas9}

Conditional replacement of the 3'UTR is possible in experimental animals, but not applicable for tissue engineering or potential therapeutic uses. Based on the above findings on the conditional 3'UTR replacement, we hypothesised that removing inhibitory sequences from the endogenous 3'UTR via 3'UTR editing using the CRISPR-Cas9 system could result in the elevation of endogenous gene expression in vivo in wild-type animals.

To test this hypothesis, we decided to delete $\sim 2.5 \mathrm{~kb}$ of the Gdnf 3'UTR central region from the Gdnf gene, which contains negative regulation sequences that destabilize Gdnf mRNA ${ }^{7}$. Guide RNAs (gRNAs) were designed to target the beginning and end of the genomic region corresponding to the Gdnf 3'UTR, removing most of the predicted microRNA (miR) binding sites, but leaving the

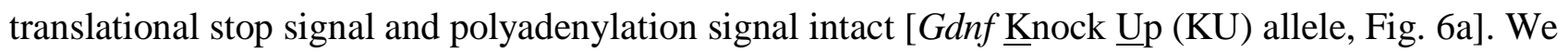
hypothesised that the resulting transcript carrying a short 3'UTR with few miR binding sites would be more stable and lead to increased GDNF levels.

Mouse zygotes were microinjected at the one-cell stage with purified gRNAs and S. pyogenes Cas9 mRNA, and embryos were dissected at E18.5 for analysis of the kidneys and brain. In embryos carrying one or two $G d n f^{K U}$ alleles (for detailed description on genotyping, please see the Methods section), the kidneys were notably smaller than kidneys from control animals (Fig. 6b) and, similar to kidneys from GDNF hypermorphs ${ }^{7}$ and $G d n f^{w t / c K U}$;Pgk1-Cre mice (Fig. 2c), displayed disorganized cortex-medulla structure (Fig. 6c). In line with these results, both Gdnf mRNA and GDNF protein levels were allele-dose-dependently increased in the kidneys of $G d n f^{w t / K U}$ and $G d n f^{K U / K U}$ mice (Fig. 6de). Furthermore, we found that Gdnf mRNA levels were also increased in the brains of $G d n f^{K U / K U}$ mice (Fig. 6f). Finally, we found that 3'UTR editing increased Gdnf mRNA expression in the human 
embryonic kidney (HEK-293) cell line (Fig 6g), suggesting that elevating endogenous gene expression via 3'UTR editing is also applicable to human cells.

a

CRISPR-Cas9 Knock Up (KU)

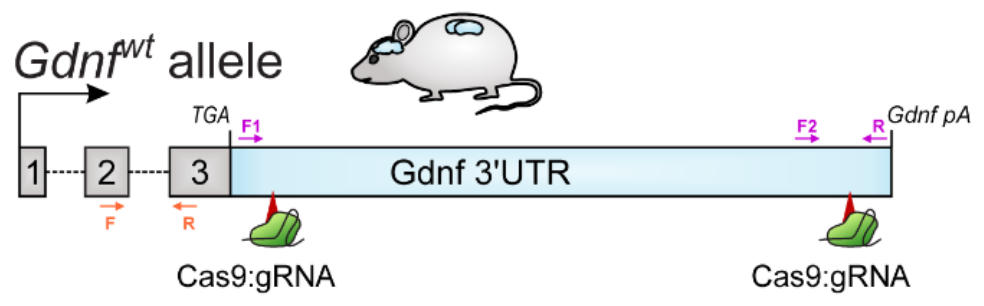

Cas9-mediated cleavage and repair b

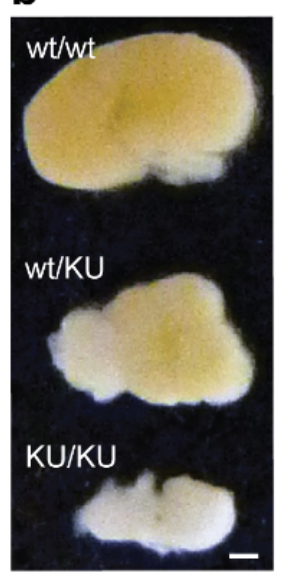

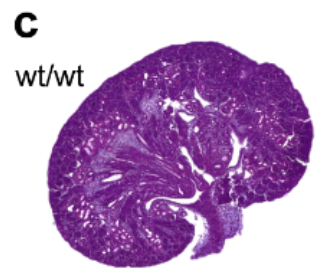

$\mathrm{wt} / \mathrm{KU}$

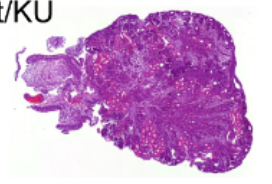

$\mathrm{KU} / \mathrm{KU}$
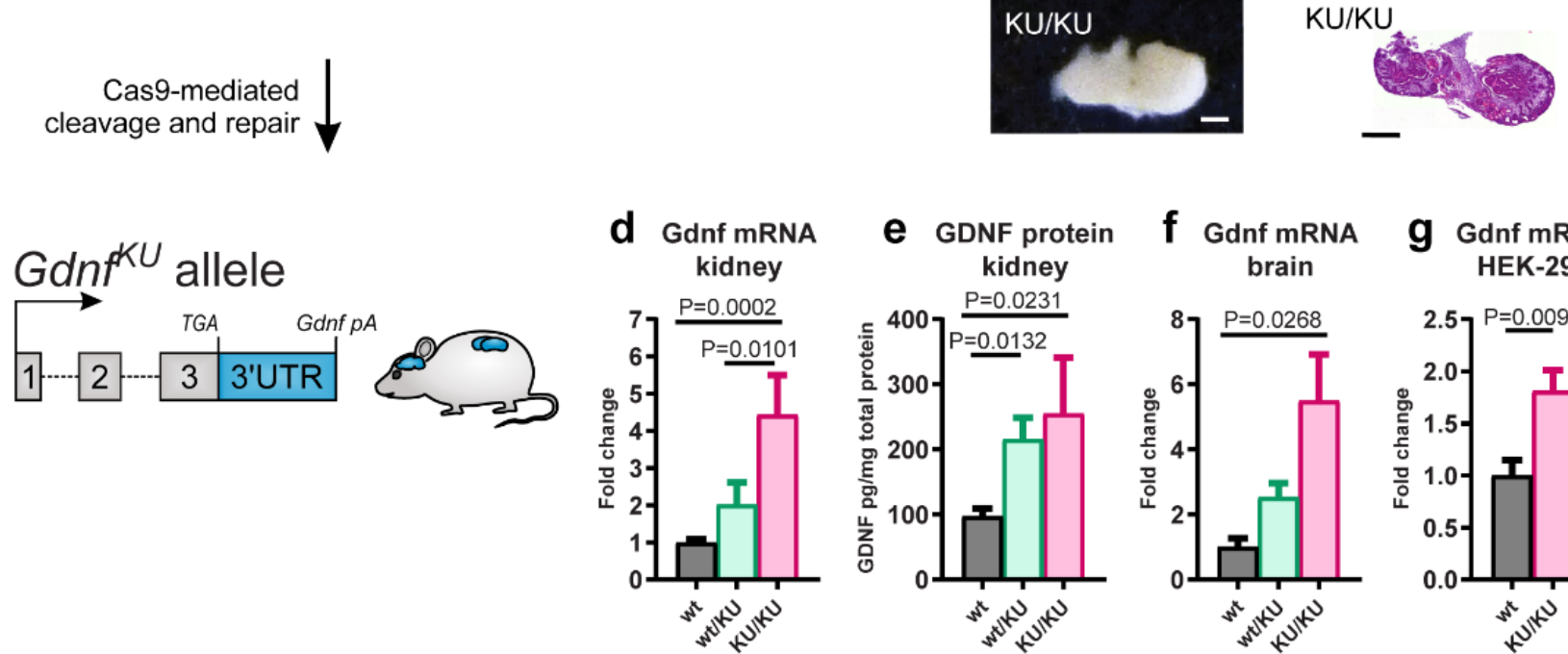

f Gdnf mRNA brain

g Gdnf mRNA
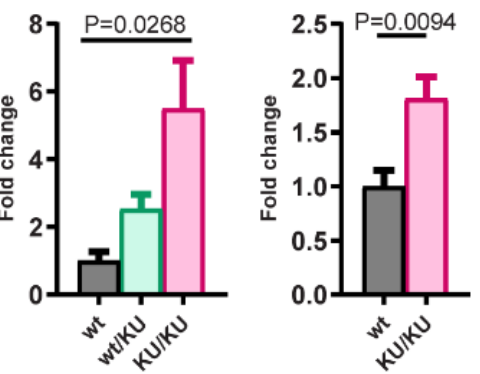

Figure 6 3'UTR editing with CRISPR-Cas9. a Schematic of gene Knock $\underline{\mathrm{Up}}(\mathrm{KU})$ via 3'UTR editing. Locations of genotyping and qPCR primers are shown in purple and orange, respectively. b-c Representative images of gross morphology (b) and histology (c) of E18.5 kidneys. d-e Gdnf mRNA (d) and protein (e) levels in the kidney. f Gdnf mRNA levels in the brain. g Gdnf mRNA levels in HEK-293 cells after 3'UTR editing. Error bars denote mean \pm SE.

Scale bar $500 \mu \mathrm{m}$. See Methods for details.

Taken together, our data suggest that excising a long 3'UTR fragment from the $G d n f$ gene increases GDNF expression in human cells and mice. Importantly, the change in GDNF levels was achieved in human cells and wild-type mice without the introduction of a foreign sequence into the genome of the animals, suggesting that endogenous gene expression can also be elevated by editing the native 3'UTR of the gene. Therefore, this approach may be useful for identifying disease pathways in in vitro models and adult animals, as well as for developing treatment strategies for human disease. 


\section{Discussion}

Currently, only $1-2 \%$ of the entire human proteome can be targeted by pharmacological agonists or activators, with the majority of targets being receptors, enzymes and transporter proteins ${ }^{39,40}$. Many of the drugs have more than one target, making it difficult to pinpoint, which of the targets are responsible for treatment or for side-effects. Therefore, a tool that enables researchers to specifically Knock Up potential drug targets one at a time would assist in identifying endogenous pathways where upregulation is advantageous in disease models.

Elevation of endogenous protein expression due to copy number variations or mutations that alter regulatory sequences of genes contributes to many human diseases, including cancers, Alzheimer's disease, Parkinson's disease, autism and schizophrenia ${ }^{37,41-45}$. Using the neurotrophic factor GDNF as a proof of concept example, we show that endogenous gene Knock Up is possible both by conditionally replacing the 3'UTR with a sequence not targeted by inhibitory factors, and by cleaving the negative regulatory regions of the native 3'UTR using the CRISPR-Cas9 system. We find no changes in celland developmental stage specific expression pattern of $G d n f$, consistent with the notion that the Knock Up approach does not alter the chromatin context of the gene. This feature makes gene Knock Up different from transgenic overexpression, where epigenetic regulation of transgene expression is often affected by the integration site of the transgene. Furthermore, gene Knock Up also differs from transcriptional activation where a nuclease dead endonuclease is fused with a transcriptional activation domain $^{46}$, because the latter, similarly to transgenic overexpression, is likely to induce ectopic gene expression in cells where the gene is not normally expressed.

We found that CNS-restricted elevation of endogenous GDNF led to several phenotypes associated with schizophrenia, including increased presynaptic dopamine levels in the striatum and SN, reduced dopamine levels in the PFC, associated changes in gene expression in the PFC, and impaired 
sensorimotor gating. Furthermore, adult-onset increase in striatal GDNF expression also reduced dopamine levels in the PFC. Several genetic association studies have implicated a link between GDNF and schizophrenia, although the results are inconclusive as yet (Extended Data Table 2). Our data suggests that a change in endogenous GDNF levels at a specific stage in development is sufficient to drive schizophrenia-like phenotypes in mice, hinting at the possibility that aberrant regulation of GDNF expression may also lead to detrimental consequences in humans.

Importantly, while GDNF Knock Up in the brain resulted in altered dopamine levels in both the striatum and prefrontal cortex, conditional knockdown of GDNF expression with the same Nestin-Cre mouse line did not alter total tissue dopamine levels in the $\mathrm{PFC}^{47}$. Therefore, the results of this study emphasise that downregulation and upregulation of endogenous gene expression do not always lead to opposite phenotypes, and thus both approaches are necessary to understand gene function.

Our results also illustrate how an increase in endogenous gene expression levels at different developmental ages can lead to different phenotypic outcomes. Mice with a constitutive increase in endogenous GDNF levels $\left(G d n f^{\text {vthyper }}\right)^{7}$ and mice with constitutively active GDNF receptor RET protooncogene signalling $\left(\operatorname{Ret}^{M E N 2 B / M E N 2 B}\right)^{48}$ have increased striatal dopamine levels, but no change in PFC dopamine levels ${ }^{49}$ (Extended Data Fig. 3e) and no deficit in $\mathrm{PPI}^{50}$. In accordance with this finding, patients with multiple endocrine neoplasia, type 2B (MEN2B), expressing constitutively active RET have not been reported to display neurological deficits or schizophrenia. At the same time, PFC dopamine levels in adult $G d n f^{c K U / c K U}$ mice are dramatically reduced two months after endogenous GDNF upregulation in the striatum even with minor changes in striatal dopamine levels (Fig 5a-d). Therefore, the time of onset of an increase in endogenous gene expression clearly affects brain development and function, and methods like gene conditional Knock Up can assist in uncovering the function of endogenous genes at specific developmental time points. 
The levels of more than one half of all protein-coding genes are predicted to be negatively regulated through the $3^{\prime} \mathrm{UTR}^{51,52}$. Therefore-as also supported by results from a recent in vitro study ${ }^{53}-3$ 'UTR editing could be used to upregulate the expression of many or most of these genes in their native chromatin context. At the same time, it is increasingly recognised that 3'UTRs can have other functions, such as regulating mRNA localization or protein-protein interactions ${ }^{54-56}$, which are likely to limit the use of the 3'UTR editing for gene Knock Up. However, once relevant motifs are identified, it could be feasible to retain the sequences required for these other functions by selecting appropriate gRNAs for Cas9-mediated excision, or by incorporating those motifs into the FLEx cassette, thus expanding the list of potential target genes for the Knock Up approach.

Taken together, we believe that the described Knock Up modes: constitutive (Pgk1-Cre), tissue specific (Nestin-Cre), inducible (Esr1-Cre), adult-onset (AAV-Cre) and CRISPR-Cas9-driven Knock Up in wild-type mice each provide new opportunities for both basic research and translational medicine. 


\section{Methods}

Animals. All animal experiments were conducted according to the $3 \mathrm{R}$ principles of the European Union Directive 2010/63/EU governing the care and use of experimental animals, and following local laws and regulations (Finnish Act on the Protection of Animals Used for Scientific or Educational Purposes (497/2013), Government Decree on the Protection of Animals Used for Scientific or Educational Purposes (564/2013)). The protocols were authorised by the national Animal Experiment Board of Finland (license numbers ESAVI/11198/04.10.07/2014 and ESAVI/12046/04.10.07/2017). Mice were maintained in a 1290la/ICR/C57bl6 mixed genetic background. The mice were grouphoused in a specific pathogen free stage with ad libitum access to food and water under a 12-h lightdark cycle (lights on at 6 a.m.) at relative humidity of 50-60\% and room temperature $21 \pm 1{ }^{\circ} \mathrm{C}$. Bedding (aspen chips, Tapvei) and nest material (Tapvei) were changed weekly and wooden blocks (Tapvei) were provided for enrichment. All behavioural tests, tissue collection and processing were performed by researchers blinded to the genotypes of the animals and in all experiments, wild-type littermates were used as controls.

Generation of $\boldsymbol{G d n f ^ { c K U }}$ knock-in allele. The targeting construct for the $G d n f^{c K U}$ allele (S Fig 1A) contained a 4021 bp 5' homologous arm spanning part of the second intron and the entire coding sequence of the mouse Gdnf gene, a 610 bp cassette containing the bovine growth hormone polyadenylation signal (bGHpA) in an inverted orientation flanked by the FLEx system ${ }^{17}$ starting $^{2}$ immediately after the stop codon, a 2615 bp Pgk1-pu $\triangle$ tk-bGHpA sequence flanked by Frt sites, and a 2927 bp 3' homologous arm. The targeting vector was synthesized by GenScript (NJ, USA) and confirmed by restriction analysis and sequencing. G4 embryonic stem cells (derived from mouse 129S6/C57BL/6Ncr) were cultured on puromycin-resistant primary embryonic fibroblast feeder layers, 
and $\sim 10^{6}$ cells were electroporated with $30 \mu \mathrm{g}$ of linearized targeting construct. After electroporation the cells were plated on 10-cm culture dishes and exposed to puromycin $(1.5 \mu \mathrm{g} / \mathrm{ml}$; Sigma). Colonies were picked after 7-9 days of selection and grown on a 96-well plate. DNA isolated from ES cells was screened by long-range PCR for both 5' and 3' homologous arms. In total, 480 clones were screened. Correct PCR products were verified by sequencing. Correctly targeted ES cells were injected into C57BL/N6 (Charles River Laboratories, MA, USA) mouse blastocysts to generate chimeric mice. Germline transmission was achieved by breeding male chimeras with C57BL/N6 females. The Pgk1pu $\Delta$ tk-bGHpA sequence was removed using CAG-Flp mouse line at F2 generation.

AAV production. Pseudotyped AAV2/5 vectors were produced using a double-transfection method with the appropriate transfer plasmid and the helper plasmid containing the essential adenoviral packaging genes, as described previously ${ }^{57}$. Vectors were purified by iodixanol step gradients and Sepharose Q column chromatography. The purified viral vector suspension was titrated with TaqMan quantitative PCR and primers targeting the WPRE sequence.

Stereotaxic surgery. Mice were anesthetized with isoflurane (3-4\% for induction and 1.5-2\% for maintenance; Oriola, Finland) in $100 \%$ oxygen. The hair on the top of the head was shaved and cleaned with Desinfektol P (Berner Pro, Finland). Mice were placed on a stereotaxic apparatus and lidocaine (Yliopiston Apteekki, Finland) was injected into the skin for local analgesia. The skin was cut with a scalpel and holes were drilled into the skull bilaterally. Two different sets of coordinates were used throughout the study: 1) A/P 1.2, M/L $\pm 2.2 \mathrm{~mm}$ and A/P 0.2, M/L $\pm 2.2 \mathrm{~mm}$, or 2) A/P 0.7, M/L \pm 2.2 , relative to the Bregma. With respect to virus diffusion and $G d n f$ expression, both injection regimens 
produced a comparable outcome. A 33G blunt NanoFil needle (World Precision Instruments, USA) was inserted into the brain parenchyma at a 10-degree angle until reaching $\mathrm{D} / \mathrm{V}-3.0 \mathrm{~mm}$, relative to Bregma. $1.0 \mu \mathrm{l}$ (injections according to the first set of coordinates) or $1.5 \mu \mathrm{l}$ (injections according to the second set of coordinates) of AAV5-Cre in Dulbecco's PBS, corresponding to $1.7 \times 10^{11}$ viral genome copies, was injected into each site at a flow rate of $0.2 \mu \mathrm{l} / \mathrm{min}$. The needle was kept in place for an additional 4-5 minutes and retracted slowly. The skin was closed with sutures and animals were subcutaneously injected with $5 \mathrm{mg} / \mathrm{kg}$ Rimadyl or Norocarp (Yliopiston Apteekki, Finland) in saline for post-operative analgesia. The mice were 3-5 months old at the time of the injections. Wild-type littermates were used as controls and were injected with the same dose of AAV5-Cre as $G d n f^{c K U}$ mice.

Tamoxifen injection. For the analysis of Gdnf mRNA and protein levels, $G d n f^{w t / c K U}$ mice were crossed to Esr1-Cre mice and pregnant females were injected i.p. with $25 \mathrm{mg} / \mathrm{kg}$ tamoxifen (Sigma-Aldrich) in corn oil on three consecutive days between E12.5-E14.5. The pups were dissected at P3 and tissues were isolated for further analysis. For RNAscope analysis, pregnant females were injected with 25 $\mathrm{mg} / \mathrm{kg}$ tamoxifen at E10.5 and tissues were harvested at E14.5.

Pre-pulse inhibition. The PPI test was performed as described previously ${ }^{50}$. Male $G d n f^{\epsilon U}{ }^{K}$ Nestin-Cre mice were analysed at an age of 3-4 months by experimenters blinded to the genotypes of the mice. Littermates were used as controls.

gRNA design and production for zygote microinjection. Guide RNAs were designed using the CRISPR Design Tool (crispr.mit.edu) $)^{58}$ and produced using overlap PCR ${ }^{59}$. The gRNA template was 
transcribed in vitro with the Megashortscript T7 Kit (AM1354, Life Technologies) by incubating 165 ng of template for 5 hours at $37^{\circ} \mathrm{C}$. In vitro transcribed RNA was purified with Megaclear Kit (AM1908, Life Technologies) and gRNA integrity was measured with Agilent 2100 Bioanalyzer (Agilent Technologies).

Two gRNAs were designed to target both the beginning $(\mathrm{A}, \mathrm{B})$ and the end $(\mathrm{C}, \mathrm{D})$ of the 3 'UTR, while leaving the translational stop signal and putative polyadenylation signal intact (Fig. 6a). All of the possible combinations $(\mathrm{A}+\mathrm{C}, \mathrm{A}+\mathrm{D}, \mathrm{B}+\mathrm{C}$ and $\mathrm{B}+\mathrm{D})$ were used for zygote injections and the efficiencies of different combinations were comparable to each other (not shown). gRNA target sequences are shown in Extended Data Table 3.

Zygote microinjection. Cas9 mRNA (5meC, $\Psi$, L-6125, Trilink Biotechnologies) and purified gRNAs were injected into the pronucleus at concentrations of $25 \mathrm{ng} / \mathrm{ml}$ and $12.5 \mathrm{ng} / \mathrm{ml}$, respectively, in an injection buffer containing $0.25 \mathrm{mM}$ EDTA and $10 \mathrm{mM}$ Tris- $\mathrm{HCl}, \mathrm{pH}$ 7.4. Prior to microinjection, the mixture was centrifuged at $13,200 \mathrm{rpm}$ for $30 \mathrm{~min}$ at $4{ }^{\circ} \mathrm{C}$ in a standard table top centrifuge and the supernatant was collected into a fresh RNase-free tube.

Isolation of tissues. Pregnant females were deeply anesthetized with $\mathrm{CO}_{2}$, followed by cervical dislocation and decapitation, and embryos were dissected at indicated time points. P3 mice were sacrificed by decapitation. The kidneys were either immersed in $4 \%$ formaldehyde (Sigma, in $1 \times$ PBS) overnight, followed by dehydration and paraffinization (Leica ASP 200), or immediately snap frozen on dry ice and stored at $-80{ }^{\circ} \mathrm{C}$ until analysed for mRNA and protein expression. Brains from E18.5 embryos were snap frozen and stored at $-80{ }^{\circ} \mathrm{C}$. 
To isolate samples for biochemical analysis, adult mice were deeply anesthetized with $\mathrm{CO}_{2}$, followed by cervical dislocation and decapitation. The brain was quickly dissected and immersed in ice-cold PBS. Brain regions of interest were isolated from 2-mm slices cut using a brain matrix (Stoelting). Tissue samples dedicated for RNA extraction or HPLC, as well as for kidney GDNF ELISA, were immediately snap frozen on dry ice and stored at $-80{ }^{\circ} \mathrm{C}$ until processed. For GDNF ELISA, brain samples were immediately lysed in lysis buffer (see below) and stored at $-80{ }^{\circ} \mathrm{C}$ until analysis.

To isolate samples for in situ hybridization, adult mice were anesthetized with pentobarbital (Mebunat, 200 mg/kg, i.p., Yliopiston Apteekki) and perfused with warm PBS and 4\% formaldehyde. Samples were post-fixed in $4 \%$ formaldehyde for 24 hours at room temperature and paraffinised or dehydrated in $30 \%$ sucrose (Thermo Fisher Scientific) in PBS prior to sectioning.

RNA and protein isolation. Total RNA was isolated using Trizol Reagent (Thermo Fisher Scientific) or RNAqueous Micro Kit (Thermo Fisher Scientific). Tissue samples for protein analysis were homogenized in lysis buffer prepared according to the recipe in the GDNF Emax Immunoassay System protocol (Promega). The tissue homogenate was centrifuged at $5000 \mathrm{rpm}$ for 15 minutes at $4{ }^{\circ} \mathrm{C}$ and the supernatant was used immediately or stored at $-80{ }^{\circ} \mathrm{C}$ until further processing. Each protein sample was only thawed and used once.

Genotyping. Genotyping samples were collected at weaning and/or during dissection. Genomic DNA was isolated from the tail using Extracta DNA Prep for PCR - tissue (Quanta Biosciences, USA). Routine genotyping was performed using AccuStart II GelTrack PCR SuperMix (Quanta Biosciences, USA) and analysed by electrophoresis using 1.5-2\% agarose gels in borate buffer. Primers used for 
genotyping the mice for the presence of $G d n f^{c K U}$ allele are indicated on Extended Data Fig. 1a and Extended Data Table 3.

Genotyping of tissues obtained from 3'UTR editing using the CRISPR-Cas9 posed a challenge due to mosaicism in analysed tissues. Because GDNF is not expressed ubiquitously, but rather in defined cell types at various stages of development, we were unable to rely on genotyping from the genomic DNA. Instead, we used cDNA synthesised using random hexamer primers as a template for genotyping, to be able to specifically detect the presence of the cleaved allele in GDNF-expressing cells. To our surprise, the genotypes in the brain and kidney obtained from the same animals did not always coincide, suggesting that Cas9-mediated DNA cleavage and repair had taken place after the cellular lineages for the particular cell types had separated. Primers used for genotyping the $G d n f^{K U}$ allele are indicated in Extended Data Table 3 and Fig. 6a.

Reverse transcription and quantitative PCR. 150 to $500 \mathrm{ng}$ of total RNA per sample were treated with RNase-free DNase I (Thermo Fisher Scientific). DNase I was inactivated with 5 mM EDTA at 65 ${ }^{\circ} \mathrm{C}$, immediately followed by a reverse transcription reaction using random hexamer primers and RevertAid Reverse Transcriptase (Thermo Fisher Scientific). Complementary DNA (cDNA) was diluted 1:10 and stored at $-20{ }^{\circ} \mathrm{C}$ until used for qPCR.

Quantitative PCR was performed with LightCycler 480 SYBR Green I Master (Roche) or with BioRad C1000 Touch Thermal Cycler upgraded to CFX384 System (BioRad), supplied with SYBR Green I Master (Roche) and 250 pmol primers, in $10 \mu$ total volume in 384-well plates. Each reaction included cDNA or a negative control (minus-reverse transcription control or water). Mouse Actb or a combination of Pgk1, Hprt and Gapdh were used as reference genes. Primer sequences are given in 
Extended Data Table 3. All samples were analysed in duplicates. Results for a biological repeat were discarded when the $C_{q}$ value for one or more of the replicates was 40 or 0 , or when the $C_{q}$ difference between replicates was $>1$.

RNA sequencing. Total RNA was quality checked on an Agilent 2200 TapeStation system. RNA quantity was measured using a NanoDrop ND-1000 Spectrophotometer and $200 \mathrm{ng}$ RNA/sample were used for library preparation using TruSeq Stranded mRNA protocol (Illumina). The yield and quality of the amplified libraries was analysed using Qubit (Thermo Fisher) and the Agilent 2200 TapeStation system. The indexed cDNA libraries were normalised and combined, and the pools were sequenced on the Illumina Nextseq for a 75 -cycle v2.5 sequencing run, generating 75 bp single-end reads. Base calling and demultiplexing was performed using CASAVA software with default settings generating Fastq files for further downstream mapping and analysis.

Fastq files were aligned to the mouse reference genome (mm10) employing the STAR aligner ${ }^{60}$. Mapped reads from the subsequent bam files were counted in annotated exons using featureCounts ${ }^{61}$. Reference genome sequence in fasta format and entrez gene annotations were retrieved from UCSC. The resulting count table was imported to R/Bioconductor and differential gene expression analysis was carried out with edgeR ${ }^{62}$. Analysis was performed on genes that had 1 count per million (cpm) in at least 3 samples and normalised with TMM normalisation. Genes with an adjusted p-value under 0.05 were termed significant and were used for GO analysis using the topGO library ${ }^{63}$. A similarity matrix was constructed of the top $150 \mathrm{GO}$ terms with the GOSemSim library ${ }^{64}$, which was then clustered by tSNE. The t-SNE result was cut into clusters using dynamic tree cutting with the dynamic TreeCut library. Plots were prepared in ggplot2. 
Analysis of protein levels. Quantification of GDNF protein levels in the brain and kidney was performed using GDNF Emax Immunoassay (Promega) with acid treatment. Due to high background in protein samples derived from the striatum, a negative control sample from $G d n f^{c K O / K O}$; Nestin-Cre mice-dissected at the same time as experimental samples and obtained from an animal of comparable age - was always included in GDNF ELISA plate. We were not able to detect a difference between wild-type and $G d n f^{K O / K O}$;Nestin-Cre samples if the brain tissue was snap frozen before lysis, therefore fresh tissue was always lysed before freezing and each frozen sample was thawed and used once. 20$100 \mu \mathrm{g}$ of total protein from the brain or kidney, measured with the DC Protein Assay (Bio-Rad), was loaded on the ELISA plate. Signal obtained from the $G d n f^{\epsilon K O / K O}$;Nestin-Cre negative control sample was subtracted from experimental striatal samples. All samples were analysed in duplicates.

HPLC. The levels of dopamine and its metabolites were analysed as previously described ${ }^{65}$, using HPLC with electrochemical detection.

Histological analysis and in situ hybridization. For histological analysis, 5- $\mu$ m paraffin sections were stained with Harris's hematoxylin (Merck) and eosin Y (Sigma-Aldrich), according to a routine protocol. RNA in situ hybridization was performed as previously described ${ }^{7}$. Briefly, fresh $5-\mu \mathrm{m}$ paraffin sections from embryonic kidney or adult brain were hybridized with RNAscope ${ }^{66}$ probes (Advanced Cell Diagnostics) detecting Gdnf mRNA, according to manufacturer's recommendations. Sections were scanned with a Pannoramic 250 digital slide scanner (3D Histech). 
Cell culture. gRNAs targeting the genomic region corresponding to human Gdnf 3'UTR were designed using the CRISPR Design Tool (crispr.mit.edu) ${ }^{58}$ and are indicated in Extended Data Table 3. The gRNAs were cloned into pAAV-EF1a-eGFP ${ }^{67}$ (a gift from Brandon Harvey). Human embryonic kidney 293 (HEK-293) cells were cultured at $37^{\circ} \mathrm{C}$ with $5 \% \mathrm{CO}_{2}$ in Dulbecco's modified Eagle’s medium (DMEM) supplemented with $10 \%$ fetal bovine serum (FBS; SV30160, Thermo Fisher Scientific, Waltham, MA, USA) and 1× Normocin (ant-nr-2, InvivoGen, San Diego, CA, USA). HEK293 cells were passaged for two consecutive days before transfection with pAAV-dgRNA-EF1a-eGFP and SpCas9-2A-Puro (a gift from Feng Zhang, Addgene plasmid \# 62988) ${ }^{68}$ plasmids using the calcium phosphate co-precipitation method. Medium was replaced $6 \mathrm{~h}$ after transfection, and cells were cultured for $48 \mathrm{~h}$ before sorting using BD Fluorescent Activated Cells Sorting (FACS) Aria II (BD Biosciences, San Jose, USA). After FACS, eGFP-positive cell population was plated in a 10-cm gelatinized plate $\left(10^{4}\right.$ cells/plate). Puromycin selection $(1 \mu \mathrm{g} / \mathrm{ml})$ was maintained for 3 days, and single cell colonies were picked and expanded for DNA and RNA isolation.

Fast-scan cyclic voltammetry. At 6-8 months of age, mice were decapitated, the brain was removed, and 300- $\mu$ m-thick coronal slices containing the striatum were cut on a model $7000 \mathrm{smz}-2$ vibratome (Campden Instruments) in oxygen-bubbled (95\% O2, 5\% CO2) ice-cold cutting artificial cerebrospinal fluid (ACSF) containing the following (in mM): $75 \mathrm{NaCl}, 2.5 \mathrm{KCl}, 26 \mathrm{NaHCO}, 1.25 \mathrm{NaH} 2 \mathrm{PO} 4,2$ $\mathrm{MgCl} 2,0.7 \mathrm{CaCl}$, and 100 glucose. The slices were allowed to recover in a holding chamber for 20 min at $34^{\circ} \mathrm{C}$, followed by $1-3 \mathrm{~h}$ at room temperature in oxygen-bubbled $(95 \% \mathrm{O} 2,5 \% \mathrm{CO} 2)$ recording ACSF containing the following (in mM): $119 \mathrm{NaCl}, 3 \mathrm{KCl}, 26 \mathrm{MgSO} 4,1 \mathrm{KH} 2 \mathrm{PO} 4,1.2 \mathrm{MgCl} 2,2$ $\mathrm{CaCl} 2$, and 10 glucose. In the recording chamber, the slices were perfused continuously with oxygenbubbled recording ACSF. Fast-scan cyclic voltammetry recordings were performed using cylindrical 5 
$\mu \mathrm{m}$ carbon fiber electrodes positioned at the dSTR $\sim 50 \mu \mathrm{m}$ below the exposed surface. Striatal slices were stimulated electrically at 2 min intervals using a stainless steel bipolar electrode placed $\sim 100 \mu \mathrm{m}$ from the recording electrode. Square pulses of $0.4 \mathrm{~ms}$ (AAV-Cre injected mice) or $1 \mathrm{~ms}$ $\left(G d n f^{c U}\right.$;Nestin-Cre mice) in duration were produced using an Iso-Flex stimulus isolator triggered by a Master-8 pulse generator (A.M.P.I.). A stimulus magnitude of $200 \mathrm{~mA}$ was obtained by selecting the minimum value that produced the maximum response reliably. Triangular voltage ramps from a holding potential of $-450 \mathrm{mV}$ to $+800 \mathrm{mV}$ over $9 \mathrm{~ms}$ (scan rate of $300 \mathrm{mV} / \mathrm{ms}$ ) were applied to the carbon fiber electrode at $100 \mathrm{~ms}$ intervals. In burst stimulus experiments, a burst of 5 pulses at $20 \mathrm{~Hz}$ was used. The current was recorded using an Axopatch 200B amplifier (Molecular Devices), filtered through a $5 \mathrm{kHz}$ low-pass Bessel filter, and digitized at $40 \mathrm{kHz}$ (ITC-18 board; InstruTech). Triangular wave generation and data acquisition were controlled and the recorded transients were characterized using a computer routine in IGOR Pro (WaveMetrics) ${ }^{69,70}$. Background-subtracted cyclic voltammograms obtained with $1 \mu \mathrm{M}$ dopamine solution (dopamine-HCl; Sigma-Aldrich) were used to calibrate the electrodes.

Statistical analyses. All values are presented as mean \pm standard error of the mean. Statistical comparison between two groups was performed using unpaired Student's $t$-test with two-tailed distribution. F-test was used to compare variances. Multiple comparisons were performed with oneway or two-way analysis of variance (ANOVA), followed by Tukey's or Sidak's post hoc test. Nonparametric data was analysed with Kruskal-Wallis test, followed by Dunn's multiple comparison analysis. Statistical analysis was performed with GraphPad Prism v8. Quantitative PCR data was analysed as described previously ${ }^{71}$, using the geometric mean of reference genes for normalisation. 
Statistical significance level was set at $p<0.05$. Details on statistical analysis are provided in Extended Data Table 4.

\section{Data availability}

The GEO database accession number for the RNA sequencing data reported in this paper will be added here prior to publishing. Other datasets in the present study are available from the corresponding author on reasonable request.

\section{Correspondence}

Correspondence and requests for materials should be addressed to Jaan-Olle Andressoo (jaanolle.andressoo@helsinki.fi) or Kärt Mätlik (kmatlik01@ rockefeller.edu).

\section{Acknowledgements}

The authors wish to thank S. Wiss and J. Lahtinen for technical assistance and A. Damdimopoulus for bioinformatics analysis of RNA sequencing data. Genetically modified mice were generated at Turku Center for Disease Modeling, and the authors thank K. Hovirinta, N. Messner, M. Niiranen, H. Niittymäki and J. Palmu for technical assistance. K.M. was supported by the doctoral program Brain and Mind, Alfred Kordelin Foundation and Sigrid Juselius Foundation. J.O.A. was supported by the Academy of Finland (grant no. 297727), Sigrid Juselius Foundation, Alzheimerfonden, Faculty of Medicine at the University of Helsinki, Helsinki Institute of Life Science and by European Research 
Council (ERC) under the European Union's Horizon 2020 research and innovation programme (grant no. 724922). G.T. was supported by the Finnish Parkinson Foundation. D.R.G. was supported by the Finnish Parkinson Foundation, Fulbright Finland and the Biomedicum Helsinki Foundation. A.P. was supported by Jane and Aatos Erkko Foundation. J.K. was supported by the Finnish Cultural Foundation and Emil Aaltonen Foundation. J.J was supported by the Swedish Research Council, the Swedish Foundation for Strategic Research, the Swedish Brain Foundation and the Swedish Government Initiative for Strategic Research Areas (MultiPark \& StemTherapy).

\section{Author contributions}

K.M. planned and designed experiments, analysed and interpreted the data, prepared figures and wrote the manuscript. K.M., S.O., D.R.G., A.M.R., G.T., L.L.P., A.P., T.P.P., J.K. and M.C.C. performed experiments. D.R.G. and A.P. designed and performed fast-scan cyclic voltammetry experiments. K.M. and N.S. analysed processed RNA sequencing data. F.Z. and P.S. generated the $G d n f^{c K U}$ allele. J.J. produced AAV. J.O.A. planned and designed experiments, interpreted the data, provided funding and contributed to writing the manuscript. All authors read and approved the manuscript.

\section{References}

1 Doyle, A., McGarry, M. P., Lee, N. A. \& Lee, J. J. The construction of transgenic and gene knockout/knockin mouse models of human disease. Transgenic Res 21, 327-349, doi:10.1007/s11248-011-9537-3 (2012).

2 Gennarino, V. A. et al. Pumilio1 haploinsufficiency leads to SCA1-like neurodegeneration by increasing wild-type Ataxin1 levels. Cell 160, 1087-1098, doi:10.1016/j.cell.2015.02.012 (2015).

3 Ross, O. A. et al. Genomic investigation of alpha-synuclein multiplication and parkinsonism. Ann Neurol 63, 743-750, doi:10.1002/ana.21380 (2008). 
4 Elia, J. et al. Genome-wide copy number variation study associates metabotropic glutamate receptor gene networks with attention deficit hyperactivity disorder. Nat Genet 44, 78-84, doi:10.1038/ng.1013 (2011).

5 Glessner, J. T. et al. Autism genome-wide copy number variation reveals ubiquitin and neuronal genes. Nature 459, 569-573, doi:10.1038/nature07953 (2009).

6 Garneau, N. L., Wilusz, J. \& Wilusz, C. J. The highways and byways of mRNA decay. Nat Rev Mol Cell Biol 8, 113-126, doi:10.1038/nrm2104 (2007).

$7 \quad$ Kumar, A. et al. GDNF Overexpression from the Native Locus Reveals its Role in the Nigrostriatal Dopaminergic System Function. PLoS Genet 11, e1005710, doi:10.1371/journal.pgen.1005710 (2015).

8 Pichel, J. G. et al. Defects in enteric innervation and kidney development in mice lacking GDNF. Nature 382, 73-76 (1996).

9 Moore, M. W. et al. Renal and neuronal abnormalities in mice lacking GDNF. Nature 382, 7679 (1996).

10 Sainio, K. et al. Glial-cell-line-derived neurotrophic factor is required for bud initiation from ureteric epithelium. Development 124, 4077-4087 (1997).

11 Sanchez, M. P. et al. Renal agenesis and the absence of enteric neurons in mice lacking GDNF. Nature 382, 70-73 (1996).

12 Lin, L. F., Doherty, D. H., Lile, J. D., Bektesh, S. \& Collins, F. GDNF: a glial cell line-derived neurotrophic factor for midbrain dopaminergic neurons. Science 260, 1130-1132 (1993).

13 Ibanez, C. F. \& Andressoo, J. O. Biology of GDNF and its receptors - Relevance for disorders of the central nervous system. Neurobiol Dis 97, 80-89, doi:10.1016/j.nbd.2016.01.021 (2017).

14 Howes, O. D. \& Kapur, S. The dopamine hypothesis of schizophrenia: version III--the final common pathway. Schizophr Bull 35, 549-562, doi:10.1093/schbul/sbp006 (2009).

15 Howes, O. D. et al. The nature of dopamine dysfunction in schizophrenia and what this means for treatment. Arch Gen Psychiatry 69, 776-786, doi:10.1001/archgenpsychiatry.2012.169 (2012).

16 McCutcheon, R. A., Abi-Dargham, A. \& Howes, O. D. Schizophrenia, Dopamine and the Striatum: From Biology to Symptoms. Trends Neurosci, doi:10.1016/j.tins.2018.12.004 (2019).

17 Schnutgen, F. et al. A directional strategy for monitoring Cre-mediated recombination at the cellular level in the mouse. Nat Biotechnol 21, 562-565, doi:10.1038/nbt811 (2003).

18 Kellendonk, C. et al. Transient and selective overexpression of dopamine D2 receptors in the striatum causes persistent abnormalities in prefrontal cortex functioning. Neuron 49, 603-615, doi:10.1016/j.neuron.2006.01.023 (2006).

19 Li, Y. C., Kellendonk, C., Simpson, E. H., Kandel, E. R. \& Gao, W. J. D2 receptor overexpression in the striatum leads to a deficit in inhibitory transmission and dopamine sensitivity in mouse prefrontal cortex. Proc Natl Acad Sci U S A 108, 12107-12112, doi:10.1073/pnas.1109718108 (2011).

20 Kakoki, M. et al. Altering the expression in mice of genes by modifying their 3' regions. Dev Cell 6, 597-606, doi:10.1016/S1534-5807(04)00094-2 (2004).

$21 \mathrm{Li}, \mathrm{H}$. et al. Development of the urogenital system is regulated via the 3'UTR of GDNF. Sci Rep 9, 5302, doi:10.1038/s41598-019-40457-1 (2019).

22 Lallemand, Y., Luria, V., Haffner-Krausz, R. \& Lonai, P. Maternally expressed PGK-Cre transgene as a tool for early and uniform activation of the Cre site-specific recombinase. Transgenic Res 7, 105-112 (1998). 
23 Hayashi, S. \& McMahon, A. P. Efficient recombination in diverse tissues by a tamoxifeninducible form of Cre: a tool for temporally regulated gene activation/inactivation in the mouse. Dev Biol 244, 305-318, doi:10.1006/dbio.2002.0597 (2002).

24 Tronche, F. et al. Disruption of the glucocorticoid receptor gene in the nervous system results in reduced anxiety. Nat Genet 23, 99-103 (1999).

25 Hidalgo-Figueroa, M., Bonilla, S., Gutierrez, F., Pascual, A. \& Lopez-Barneo, J. GDNF is predominantly expressed in the PV+ neostriatal interneuronal ensemble in normal mouse and after injury of the nigrostriatal pathway. $J$ Neurosci 32, 864-872, doi:10.1523/JNEUROSCI.2693-11.2012 (2012).

26 Berke, J. D. Functional properties of striatal fast-spiking interneurons. Front Syst Neurosci $\mathbf{5}$, 45, doi:10.3389/fnsys.2011.00045 (2011).

27 Slifstein, M. et al. Deficits in prefrontal cortical and extrastriatal dopamine release in schizophrenia: a positron emission tomographic functional magnetic resonance imaging study. JAMA Psychiatry 72, 316-324, doi:10.1001/jamapsychiatry.2014.2414 (2015).

28 Zhan, L. et al. Altered expression and coregulation of dopamine signalling genes in schizophrenia and bipolar disorder. Neuropathol Appl Neurobiol 37, 206-219, doi:10.1111/j.1365-2990.2010.01128.x (2011).

29 Zhang, Y. et al. Polymorphisms in human dopamine D2 receptor gene affect gene expression, splicing, and neuronal activity during working memory. Proc Natl Acad Sci U S A 104, 2055220557, doi:10.1073/pnas.0707106104 (2007).

30 Bertolino, A. et al. Functional variants of the dopamine receptor D2 gene modulate prefrontostriatal phenotypes in schizophrenia. Brain 132, 417-425, doi:10.1093/brain/awn248 (2009).

31 Bolkan, S. S., Carvalho Poyraz, F. \& Kellendonk, C. Using human brain imaging studies as a guide toward animal models of schizophrenia. Neuroscience 321, 77-98, doi:10.1016/j.neuroscience.2015.05.055 (2016).

32 Koch, M. \& Bubser, M. Deficient sensorimotor gating after 6-hydroxydopamine lesion of the rat medial prefrontal cortex is reversed by haloperidol. Eur J Neurosci 6, 1837-1845 (1994).

33 Swerdlow, N. R. et al. Forebrain D1 function and sensorimotor gating in rats: effects of D1 blockade, frontal lesions and dopamine denervation. Neurosci Lett 402, 40-45, doi:10.1016/j.neulet.2006.03.060 (2006).

34 Siuta, M. A. et al. Dysregulation of the norepinephrine transporter sustains cortical hypodopaminergia and schizophrenia-like behaviors in neuronal rictor null mice. PLoS Biol 8, e1000393, doi:10.1371/journal.pbio.1000393 (2010).

35 Braff, D. et al. Prestimulus effects on human startle reflex in normals and schizophrenics. Psychophysiology 15, 339-343 (1978).

36 Kohl, S., Heekeren, K., Klosterkotter, J. \& Kuhn, J. Prepulse inhibition in psychiatric disorders-apart from schizophrenia. J Psychiatr Res 47, 445-452, doi:10.1016/j.jpsychires.2012.11.018 (2013).

37 de Lacy, N. \& King, B. H. Revisiting the relationship between autism and schizophrenia: toward an integrated neurobiology. Annu Rev Clin Psychol 9, 555-587, doi:10.1146/annurevclinpsy-050212-185627 (2013).

38 Bakken, T. E. et al. A comprehensive transcriptional map of primate brain development. Nature 535, 367-375, doi:10.1038/nature18637 (2016).

39 Rask-Andersen, M., Almen, M. S. \& Schioth, H. B. Trends in the exploitation of novel drug targets. Nat Rev Drug Discov 10, 579-590, doi:10.1038/nrd3478 (2011).

40 Santos, R. et al. A comprehensive map of molecular drug targets. Nat Rev Drug Discov 16, 1934, doi:10.1038/nrd.2016.230 (2017). 
41 Mayr, C. \& Bartel, D. P. Widespread shortening of 3'UTRs by alternative cleavage and polyadenylation activates oncogenes in cancer cells. Cell 138, 673-684, doi:10.1016/j.cell.2009.06.016 (2009).

42 Rovelet-Lecrux, A. et al. APP locus duplication causes autosomal dominant early-onset Alzheimer disease with cerebral amyloid angiopathy. Nat Genet 38, 24-26, doi:10.1038/ng1718 (2006).

43 Ibanez, P. et al. Causal relation between alpha-synuclein gene duplication and familial Parkinson's disease. Lancet 364, 1169-1171, doi:10.1016/S0140-6736(04)17104-3 (2004).

44 Chartier-Harlin, M. C. et al. Alpha-synuclein locus duplication as a cause of familial Parkinson's disease. Lancet 364, 1167-1169, doi:10.1016/S0140-6736(04)17103-1 (2004).

45 Cooper, D. N., Krawczak, M., Polychronakos, C., Tyler-Smith, C. \& Kehrer-Sawatzki, H. Where genotype is not predictive of phenotype: towards an understanding of the molecular basis of reduced penetrance in human inherited disease. Hum Genet 132, 1077-1130, doi:10.1007/s00439-013-1331-2 (2013).

46 Sander, J. D. \& Joung, J. K. CRISPR-Cas systems for editing, regulating and targeting genomes. Nat Biotechnol 32, 347-355, doi:10.1038/nbt.2842 (2014).

47 Kopra, J. et al. GDNF is not required for catecholaminergic neuron survival in vivo. Nat Neurosci 18, 319-322, doi:10.1038/nn.3941 (2015).

48 Mijatovic, J. et al. Constitutive Ret activity in knock-in multiple endocrine neoplasia type B mice induces profound elevation of brain dopamine concentration via enhanced synthesis and increases the number of TH-positive cells in the substantia nigra. J Neurosci 27, 4799-4809, doi:10.1523/JNEUROSCI.5647-06.2007 (2007).

49 Kopra, J. et al. Constitutive Ret signaling leads to long-lasting expression of amphetamineinduced place conditioning via elevation of mesolimbic dopamine. Neuropharmacology 128, 221-230, doi:10.1016/j.neuropharm.2017.10.010 (2018).

50 Matlik, K., Voikar, V., Vilenius, C., Kulesskaya, N. \& Andressoo, J. O. Two-fold elevation of endogenous GDNF levels in mice improves motor coordination without causing side-effects.

Sci Rep 8, 11861, doi:10.1038/s41598-018-29988-1 (2018).

51 Friedman, R. C., Farh, K. K., Burge, C. B. \& Bartel, D. P. Most mammalian mRNAs are conserved targets of microRNAs. Genome research 19, 92-105, doi:10.1101/gr.082701.108 (2009).

52 Khabar, K. S. The AU-rich transcriptome: more than interferons and cytokines, and its role in disease. J Interferon Cytokine Res 25, 1-10, doi:10.1089/jir.2005.25.1 (2005).

53 Zhao, W. \& Erle, D. J. Widespread Effects of Chemokine 3' Untranslated Regions on mRNA Degradation and Protein Production in Human Cells. J Immunol 201, 1053-1061, doi:10.4049/jimmunol.1800114 (2018).

54 Mayr, C. Evolution and Biological Roles of Alternative 3'UTRs. Trends Cell Biol 26, 227-237, doi:10.1016/j.tcb.2015.10.012 (2016).

55 Ma, W. \& Mayr, C. A Membraneless Organelle Associated with the Endoplasmic Reticulum Enables 3'UTR-Mediated Protein-Protein Interactions. Cell 175, 1492-1506 e1419, doi:10.1016/j.cell.2018.10.007 (2018).

56 Mayr, C. What Are 3' UTRs Doing? Cold Spring Harb Perspect Biol, doi:10.1101/cshperspect.a034728 (2018).

57 Ulusoy, A., Sahin, G., Bjorklund, T., Aebischer, P. \& Kirik, D. Dose optimization for long-term rAAV-mediated RNA interference in the nigrostriatal projection neurons. Mol Ther 17, 15741584, doi:10.1038/mt.2009.142 (2009). 
58 Hsu, P. D. et al. DNA targeting specificity of RNA-guided Cas9 nucleases. Nat Biotechnol 31, 827-832, doi:10.1038/nbt.2647 (2013).

59 Bassett, A. R., Tibbit, C., Ponting, C. P. \& Liu, J. L. Highly efficient targeted mutagenesis of Drosophila with the CRISPR/Cas9 system. Cell Rep 4, 220-228, doi:10.1016/j.celrep.2013.06.020 (2013).

60 Dobin, A. et al. STAR: ultrafast universal RNA-seq aligner. Bioinformatics 29, 15-21, doi:10.1093/bioinformatics/bts635 (2013).

61 Liao, Y., Smyth, G. K. \& Shi, W. featureCounts: an efficient general purpose program for assigning sequence reads to genomic features. Bioinformatics 30, 923-930, doi:10.1093/bioinformatics/btt656 (2014).

62 McCarthy, D. J., Chen, Y. \& Smyth, G. K. Differential expression analysis of multifactor RNASeq experiments with respect to biological variation. Nucleic Acids Res 40, 4288-4297, doi:10.1093/nar/gks042 (2012).

63 Alexa, A., Rahnenfuhrer, J. \& Lengauer, T. Improved scoring of functional groups from gene expression data by decorrelating GO graph structure. Bioinformatics 22, 1600-1607, doi:10.1093/bioinformatics/btl140 (2006).

$64 \mathrm{Yu}, \mathrm{G}$. et al. GOSemSim: an R package for measuring semantic similarity among GO terms and gene products. Bioinformatics 26, 976-978, doi:10.1093/bioinformatics/btq064 (2010).

65 Valros, A. et al. Evidence for a link between tail biting and central monoamine metabolism in pigs (Sus scrofa domestica). Physiol Behav 143, 151-157, doi:10.1016/j.physbeh.2015.02.049 (2015).

66 Wang, F. et al. RNAscope: a novel in situ RNA analysis platform for formalin-fixed, paraffinembedded tissues. J Mol Diagn 14, 22-29, doi:10.1016/j.jmoldx.2011.08.002 (2012).

67 Savolainen, M. H. et al. The beneficial effect of a prolyl oligopeptidase inhibitor, KYP-2047, on alpha-synuclein clearance and autophagy in A30P transgenic mouse. Neurobiol Dis 68, 1-15, doi:10.1016/j.nbd.2014.04.003 (2014).

68 Ran, F. A. et al. Genome engineering using the CRISPR-Cas9 system. Nat Protoc 8, 22812308, doi:10.1038/nprot.2013.143 (2013).

69 Mosharov, E. V. \& Sulzer, D. Analysis of exocytotic events recorded by amperometry. Nat Methods 2, 651-658, doi:10.1038/nmeth782 (2005).

70 Mosharov, E. V. Analysis of single-vesicle exocytotic events recorded by amperometry. Methods Mol Biol 440, 315-327, doi:10.1007/978-1-59745-178-9_24 (2008).

71 Varendi, K., Kumar, A., Harma, M. A. \& Andressoo, J. O. miR-1, miR-10b, miR-155, and miR-191 are novel regulators of BDNF. Cell Mol Life Sci 71, 4443-4456, doi:10.1007/s00018014-1628-x (2014). 